\title{
Retraction: A photon thermal diode
}

\author{
Zhen Chen, Carlaton Wong, Sean Lubner, Shannon Yee, John Miller, Wanyoung Jang, Corey Hardin, \\ Anthony Fong, Javier E. Garay \& Chris Dames
}

Nature Communications 5:5446 doi: 10.1038/ncomms6446 (2014); Published 17 Nov 2014; Updated 21 Aug 2017

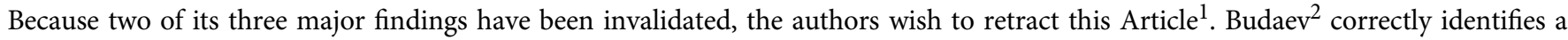
fundamental symmetry error in the way the 'inelastic thermal collimation' was configured in several crucial experiments of ref. 1, specifically the results presented in Fig. 3c (the three filled and four striped bars, labeled 'Col. 1' and 'Col. 2', respectively) and Fig. 4. As detailed in the accompanying Correspondence ${ }^{3}$, this error originated from a faulty thermal estimate ${ }^{4}$, and further modelling ${ }^{3,4}$ now confirms the problem. Although we continue to believe the heat flow measurements in ref. 1 were accurate for all of the configurations presented, due to the symmetry error none of those experimental configurations were actually relevant for two of the most important findings of the paper, which are therefore retracted: firstly, that a photon thermal diode was experimentally demonstrated, and secondly, that the 'inelastic thermal collimation' mechanism is a suitable nonlinearity for realizing thermal rectification when combined with asymmetric scattering structures (e.g., copper pyramids or etched triangular pores in silicon).

The symmetry error ${ }^{2}$ does not apply to the experiments without thermal collimation, specifically the results presented in Fig. $3 c$ for photons (the six leftmost, unfilled bars) and Supplementary Figure 12 for phonons. Therefore, the last major conclusion of the Article ${ }^{1}$ remains well-supported by those original experiments: Asymmetric scattering alone is insufficient to achieve thermal rectification.

\section{References}

1. Chen, Z. et al. A photon thermal diode. Nat. Commun. 5, 5446 (2014).

2. Budaev, B. Correspondence: The experimental requirements for a photon thermal diode. Nat. Commun. 8, 16135 (2017).

3. Chen, Z. et al. Correspondence: Reply to 'The experimental requirements for a photon thermal diode'. Nat. Commun. 8, 16136 (2017).

4. Dames, C. \& Chen, Z. Additional details about the thermal design error in 'A Photon Thermal Diode'. Preprint at http://arxiv.org/abs/1705.10902 (2017).

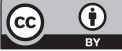

Open Access This article is licensed under a Creative Commons Attribution 4.0 International License, which permits use, sharing, adaptation, distribution and reproduction in any medium or format, as long as you give appropriate credit to the original author(s) and the source, provide a link to the Creative Commons license, and indicate if changes were made. The images or other third party material in this article are included in the article's Creative Commons license, unless indicated otherwise in a credit line to the material. If material is not included in the article's Creative Commons license and your intended use is not permitted by statutory regulation or exceeds the permitted use, you will need to obtain permission directly from the copyright holder. To view a copy of this license, visit http://creativecommons.org/licenses/by/4.0/

(C) The Author(s) 2017 